\title{
Zero-contrast percutaneous coronary interventions to preserve kidney function in patients with severe renal impairment and hemodialysis subjects
}

\author{
Jerzy Sacha ${ }^{1,2}$, Marek Gierlotka ${ }^{1}$, Przemysław Lipski ${ }^{1}$, Piotr Feusette $^{1}$, Dariusz Dudek ${ }^{3}$ \\ ${ }^{1}$ Department of Cardiology, University Hospital, Faculty of Natural Sciences and Technology, University of Opole, Opole, Poland \\ ${ }^{2}$ Faculty of Physical Education and Physiotherapy, Opole University of Technology, Opole, Poland \\ ${ }^{3}$ Institute of Cardiology, Jagiellonian University Medical College, Krakow, Poland
}

Adv Interv Cardiol 2019; 15, 2 (56): 137-142

DOI: https://doi.org/10.5114/aic.2019.86008

\begin{abstract}
A bstract
Introduction: Zero-contrast percutaneous coronary intervention (zero-PCI) is a new method for prevention of contrast-induced acute kidney injury (AKI) in patients with chronic kidney disease (CKD). However, evidence for its feasibility, safety and clinical utility is limited to reports of single cases or series of patients.

Aim: To present outcomes of zero-PCI in patients with severe CKD, including hemodialysis subjects, who were treated with this procedure in order to preserve their renal function.

Material and methods: Twenty-nine zero-PCls were performed, mostly as a staged procedure, in 20 patients with advanced CKD. In this group, 4 patients were treated with hemodialysis but presented preserved residual renal function. The estimated median risk for contrast-induced AKI in non-dialysis patients was 26\% (26-57\%).

Results: Zero-PCI was feasible in each intended patient, including those with complex left main stenosis or lesion within a saphenous vein graft, and there was no specific complication associated with this technique. After the procedure, the factual AKI prevalence was $10 \%$ and no patient required renal replacement therapy. Three of 4 hemodialysis patients preserved their residual renal function. During the median follow-up of 3.2 (1.2-5.3) months no patient experienced an acute coronary event or required revascularization.

Conclusions: Zero-PCI is a safe and promising method to preserve renal function in patients with CKD and hemodialysis patients. Such an approach is feasible even in complex coronary lesions and yields good clinical outcomes in mid-term observation.
\end{abstract}

Key words: renal insufficiency, contrast-induced nephropathy, acute kidney injury, zero-contrast percutaneous coronary intervention.

Su m m a ry

Zero-contrast percutaneous coronary intervention (zero-PCI) is a new strategy for prevention of contrast-induced acute kidney injury (AKI). This study presents outcomes of 29 zero-PCls performed in 20 patients with severe chronic kidney disease. Zero-PCI was feasible in each intended patient and there was no specific complication associated with this technique. The estimated median risk of AKI of $26 \%$ (26-57\%) before zero- $\mathrm{PCI}$ dropped to the observed prevalence of $10 \%$, and during the median follow-up of $3.2(1.2-5.3)$ months no patient experienced an acute coronary event or required revascularization.

\section{Introduction}

Contrast-induced acute kidney injury (AKI) is a severe complication of percutaneous coronary intervention $(\mathrm{PCl})$ in patients with chronic kidney disease (CKD) and is responsible for various adverse outcomes such as deterioration of renal function, necessity of dialysis, prolonged hospitalization and increased mortality [1, 2]. The risk of AKI is particularly high in CKD patients with multiple comorbidities including diabetes mellitus, congestive heart failure, hemodynamic instability, reduced plasma volume and anemia $[3,4]$. Since the incidence of AKI increases almost linearly with the amount of contrast, every method allowing contrast volume reduction

Corresponding author:

Jerzy Sacha MD, PhD, Department of Cardiology, University Hospital, Faculty of Natural Sciences and Technology, University of Opole, Al. Witosa 26, 45-401 Opole, Poland, phone: +48 7745206 60, e-mail: sacha@op.pl

Received: 12.01.2019, accepted: 7.04.2019. 
may protect renal function [2, 3]. Zero-contrast $\mathrm{PCl}$ (zero- $\mathrm{PCl}$ ) is a new emerging method for the prevention of AKI among patients with CKD [5]. However, despite initial promising results, experiences in this approach are still limited to single cases or series of patients [5-14]. Moreover, it is not known if such an approach plays any positive role in preserving residual renal function in hemodialysis patients. Since the procedure without contrast administration is demanding and may be associated with some potential complications, more evidence for its feasibility, safety and clinical utility is needed before it will be accepted as a daily practice by a wide interventional community.

\section{Aim}

The aim of this retrospective analysis was to present outcomes of zero-PCl in patients with severe CKD, including hemodialysis subjects, who underwent this procedure in order to preserve their renal function.

\section{Material and methods}

Patients with renal failure requiring percutaneous revascularization were treated with zero- $\mathrm{PCl}$ if they met one of the following criteria: (i) their estimated glomerular filtration rate (eGFR, by the Modification of Diet in Renal Disease equation) was less than $45 \mathrm{ml} / \mathrm{min} / 1.73 \mathrm{~m}^{2}$; (ii) they previously experienced contrast-induced AKI; or (iii) they were treated with hemodialysis but presented preserved residual renal function defined as urine output $\geq 500 \mathrm{ml} /$ day [15]. The main exclusion criteria were: ST-segment elevation myocardial infarction (STEMI), shock, chronic total occlusion and terminal chronic renal failure with no residual diuresis. However, patients after recent STEMI who required further revascularization and $\mathrm{AKI}$ prevention were also qualified for zero-PCI as a staged procedure. Patients with non-ST-segment elevation myocardial infarction (NSTEMI) were suitable for such an intervention if their infarct-related artery was patent without obvious thrombus on the initial coronary angiography. Previous coronary artery bypass grafting ( $C A B G)$ and the need for rotational atherectomy in non-tortuous artery were not exclusion criteria. Individual risk of AKI before zero-PCI was estimated according to the Mehran risk score with the assumption that contrast dye is not used [3]. Contrast-induced AKI was defined as an increase in the serum creatinine level of more than $0.5 \mathrm{mg} / \mathrm{dl}(44 \mu \mathrm{mol} / \mathrm{l})$ or an increase of at least $25 \%$ in the level from baseline within $72 \mathrm{~h}$ after the procedure [1].

Stable patients with known significant renal impairment underwent ultra-low contrast coronary angiography where the intended maximum contrast volume was pre-defined as less than or equal to $15 \mathrm{ml}$-details on how to perform such an examination have been summarized elsewhere [16]. However, unstable patients with acute coronary syndrome usually first underwent standard interventions and then, if they needed further revascularization, zero-PCl was performed as a staged procedure. For zero- $\mathrm{PCl}$, coronary angiography was analyzed in detail and the suitable angiographic images were displayed alongside the active fluoroscopy screen as a reference - the guidewires were inserted according to these reference images. The procedure was guided by intravascular ultrasound (IVUS), i.e. IVUS identified the lesion length, determined balloon and stent diameters, and landing zones for stent implantation, as well as verified and documented the final PCl effect. Landing zones were determined at the first non-diseased vessel segments close to the lesion or at segments with plaque burden less than $50 \%$ in cases with diffuse lesions. The reference diameters for selection of balloons and stents were determined by the maximum lumen diameter of the distal landing zone - if another stent had to be proximally placed, the proximal landing zones was a reference. The stent length was based on the distance between the distal and proximal landing zones and was measured with a calibrated Volcano IVUS probe. The $\mathrm{PCI}$ result was assessed in IVUS by calculating the stent expansion which corresponded to the ratio of the minimum stent area over the mean of the proximal and distal stent reference lumen areas multiplied by $100 \%$ (percentage). A detailed description of the zero-contrast method and its procedural options may be found elsewhere [16]. Due to legal issues, at the end of procedure, a single injection of small contrast volume (usually $5 \mathrm{ml}$ ) was performed in each patient to document the angiographic $\mathrm{PCI}$ result and lack of complications (e.g. distal perforation or embolization). Other indications for contrast injection during the intervention included: chest pain, persistent drop in blood pressure, new electrocardiographic changes and any suspicion of complications. To become familiar with this technique, operators had to undergo a training program which relied on the guidewire insertion and balloon as well as stent positioning without contrast usage during several standard PCls (i.e. under ultimate control of contrast injections) and then, for maintenance of the skill, they repeated such a procedure periodically.

All patients provided their informed written consent for the procedure and the treatment strategy was accepted by the institutional review board.

\section{Statistical analysis}

Continuous variables were presented as mean \pm standard deviation or median and interquartile range where it was appropriate according to normality tests. Categorical variables were presented as numeric values and percentages. Differences in renal function parameters before and after zero-PCI were compared using Student's paired $t$-test or the Wilcoxon signed-rank test. The threshold probability of $p<0.05$ was taken as the level of statisti- 
cal significance. All analyses were performed using NCSS 12 Statistical Software (2018). NCSS, LLC. Kaysville, Utah, USA, ncss.com/software/ncss.

\section{Results}

The study group consisted of 20 patients aged 73.7 \pm 12.8 years with severe renal impairment (eGFR 24.8 $\pm 12.5 \mathrm{ml} / \mathrm{min} / 1.73 \mathrm{~m}^{2}$ ) who underwent zero- $\mathrm{PCl}$ for prevention of AKI - 4 of these subjects were chronically treated with hemodialysis. Thirteen (65\%) patients were admitted due to acute coronary syndromes, i.e. 3 (15\%) with STEMI, 6 (30\%) with NSTEMI and 4 (20\%) with unstable angina. Three (15\%) patients were hospitalized because of heart failure, 2 (10\%) due to sustained ventricular tachycardia and 2 (10\%) were admitted for elective $\mathrm{PCl}$. Table I presents patients' characteristics. Before zero-PCl, ultra-low contrast coronary angiography was performed in 9 patients including hemodialysis subjects (median contrast volume: $13(11-24) \mathrm{ml})$. In 2 patients with advanced CKD, who were admitted for elective intervention, coronary angiography was performed during previous hospitalization. However, 9 unstable patients with acute coronary syndrome first underwent standard coronary angiography and $\mathrm{PCl}$ of the infarct related artery with median contrast volume of $150(70-200) \mathrm{ml}$, and consequently two of them developed contrast-induced AKI requiring temporal hemodialysis - since they all required further revascularization, staged zero-PCI was performed after renal function stabilization.

In general, zero-PCl was performed within 29 coronary arteries in 20 patients with advanced CKD. Sixteen of these subjects underwent staged procedures with a median time of $6(5-8)$ days after the first intervention. Multi-vessel zero-PCl was done in 7 cases. One patient with severe calcified lesions was treated with rotational atherectomy but another presenting obstruction within the saphenous vein graft required insertion of a distal protection device - both procedures were done without contrast injection.

After each intervention, due to legal issues, a small amount of contrast dye was injected to confirm the final result and exclude complications (median contrast volume: 5 (3.5-9) $\mathrm{ml}$ ). Procedural data concerning zero-PCl are presented in Table II.

According to the Mehran risk score, the estimated median risk for contrast-induced AKI before zero- $\mathrm{PCI}$ was $26 \%(26-57 \%)$ in non-dialysis patients. The creatinine and eGFR levels did not differ significantly before and after the intervention (mean change: $0.1 \pm 0.31 \mathrm{mg} / \mathrm{dl}$, $p=0.2$; and $-0.7 \pm 10.9 \mathrm{ml} / \mathrm{min} / 1.73 \mathrm{~m}^{2}, p=0.8$, respectively). However, in 2 patients the creatinine value slightly exceeded the pre-defined threshold for AKI after zero- $\mathrm{PCl}$; hence the AKI prevalence turns out to be $10 \%$. Importantly, 2 patients who previously developed contrast-induced AKI requiring hemodialysis after standard
Table I. Patients' characteristics

\begin{tabular}{|c|c|}
\hline Parameter & Overall group \\
\hline \multicolumn{2}{|l|}{ Clinical characteristics: } \\
\hline Age [years] & $73.7 \pm 12.8$ \\
\hline Males & $12(60)$ \\
\hline ACS & $13(65)$ \\
\hline Hemodialysis patients & $4(20)$ \\
\hline Diabetes mellitus & $13(65)$ \\
\hline Hypertension & $16(80)$ \\
\hline $\mathrm{CHF}$ & $16(80)$ \\
\hline VHD & $5(25)$ \\
\hline LVEF (\%) & $42.2 \pm 12.8$ \\
\hline Previous MI & $2(10)$ \\
\hline Previous $\mathrm{PCl}$ & $1(5)$ \\
\hline Previous CABG & $3(15)$ \\
\hline Dyslipidemia & $7(35)$ \\
\hline Current smoking & $1(5)$ \\
\hline \multicolumn{2}{|l|}{ Baseline laboratory values: } \\
\hline Troponin T & $202.8(89.5-995.5)$ \\
\hline $\mathrm{WBC}\left[\times 10^{3} / \mu \mathrm{l}\right]$ & $8.1(7.2-12.2)$ \\
\hline $\operatorname{RBC}\left[\times 10^{6} / \mu \mathrm{l}\right]$ & $3.9 \pm 0.8$ \\
\hline Hemoglobin [g/dl] & $11.6 \pm 2.3$ \\
\hline $\mathrm{HCT}(\%)$ & $35.9 \pm 6.7$ \\
\hline $\mathrm{PLT}\left[\times 10^{3} / \mu \mathrm{ll}\right]$ & $225(184-257)$ \\
\hline Creatinine $[\mathrm{mg} / \mathrm{dl}]$ & $3.2 \pm 1.9$ \\
\hline eGFR $\left[\mathrm{ml} / \mathrm{min} / 1.73 \mathrm{~m}^{2}\right]$ & $24.8 \pm 12.5$ \\
\hline \multicolumn{2}{|l|}{ Coronary artery disease: } \\
\hline One-vessel disease & $0(0)$ \\
\hline Two-vessel disease & $7(35)$ \\
\hline Three-vessel disease & $13(65)$ \\
\hline Left main disease & $1(5)$ \\
\hline Bypass graft disease & $2(10)$ \\
\hline
\end{tabular}

Values are $n(\%)$, mean $\pm S D$ or median (Q1-Q3). ACS - acute coronary syndrome, CHF - congestive heart failure, VHD - valvular heart disease, LVEF - left ventricular ejection fraction, $\mathrm{MI}$ - myocardial infarction, $\mathrm{PCl}$ - percutaneous coronary intervention, $C A B G$ - coronary artery bypass graft, WBC - white blood cells, RBC - red blood cells, HCT - hematocrit, PLT - platelets, eGFR - estimated glomerular filtration rate. Coronary artery disease was recognized if the diameter stenosis was at least $50 \%$

coronary angiography and $\mathrm{PCl}$ did not experience AKI after zero-PCI. In 1 patient, the troponin T value exceeded the level for type 4A myocardial infarction, yet without clinical consequences (IVUS and the final single contrast 
administration did not reveal abnormalities) [17]. In 1 patient with NSTEMI, the final small contrast injection revealed distal embolization which was subsequently treated with anticoagulants and antiplatelet agents. During the median follow-up period of $3.2(1.2-5.3)$ months, one woman with severe pulmonary hypertension died after 6 months due to right ventricular heart failure - death not related to the procedure.

Four hemodialysis patients had a median diuresis of 900 (763-1150) ml/day before and 875 (263-1000) ml/ day after the intervention $(p=0.8)$, and 3 of them preserved their residual renal function after zero- $\mathrm{PCl}$. In one of these patients, who underwent a 3-vessel zero-PCI,

\section{Table II. Zero-contrast PCI}

\begin{tabular}{lc}
\hline Procedural data & Overall group \\
\hline $\begin{array}{l}\text { Number of vessels treated with } \\
\text { zero-contrast PCI }\end{array}$ & 29 \\
\hline Staged PCI & $24(83)$ \\
\hline $\begin{array}{l}\text { Time between coronary angiography } \\
\text { and staged PCI [days] }\end{array}$ & $6(5-8)$ \\
\hline Left main artery & $1(3)$ \\
\hline Left anterior descending artery & $11(38)$ \\
\hline Left circumflex artery & $10(34)$ \\
\hline Right coronary artery & $6(21)$ \\
\hline Saphenous vein graft & $1(3)$ \\
\hline Diameter stenosis (\%) & $85.3 \pm 8.7$ \\
\hline Lesion length [mm] & $35.4 \pm 19.6$ \\
\hline Lesion area [mm²] & $2.9(2.6-3.4)$ \\
\hline Lesion plaque burden (\%) & $76(70-79)$ \\
\hline Minimal stent area [mm²] & $6.5(5.3-7.6)$ \\
\hline Stent expansion (\%) & $95(88-103)$ \\
\hline Guide wires & $2(1-2)$ \\
\hline Number of stents & $2(1-2)$ \\
\hline Total stent length [mm] & $42.9 \pm 19.7$ \\
\hline Stent diameter [mm] & $3(2.5-3.5)$ \\
\hline Pre-dilation & $42(88)$ \\
\hline Post-dilation & $41(85)$ \\
\hline Rotational atherectomy & $1(5)$ \\
\hline Distal embolic protection & $1(5)$ \\
\hline Procedure time [min] & $69-9)$ \\
\hline Radiation dose [mGy] & \\
\hline Final contrast injection [ml] & \\
\hline
\end{tabular}

Values are $n(\%)$, mean $\pm S D$ or median $(Q 1-Q 3)$. PCI-percutaneous coronary intervention. Pre-dilation and post-dilation relate to each implanted stent (total number of stents is 48). the renal function improved within some weeks, i.e. the diuresis increased from 800 to $1000 \mathrm{ml} /$ day and the rate of dialyses was reduced from 3 to 2 times per week. Another patient lost the residual renal function within several weeks after hospitalization, i.e. his diuresis dropped from 1000 to $100 \mathrm{ml} /$ day. Table III presents the summary of patients' outcomes and medications.

Table III. Outcomes of zero-contrast PCI and discharge medications

\begin{tabular}{|c|c|}
\hline Variable & Overall group \\
\hline \multicolumn{2}{|l|}{ Hospitalization: } \\
\hline Change in creatinine [mg/dl] & $0.1 \pm 0.31$ \\
\hline Change in eGFR [ml/min/1.73 $\left.\mathrm{m}^{2}\right]$ & $-0.7 \pm 10.9$ \\
\hline $\mathrm{AKI}$ after zero-contrast $\mathrm{PCl}$ & $2(10)$ \\
\hline $\begin{array}{l}\text { Renal replacement therapy } \\
\text { (non-dialysis patients) }\end{array}$ & $0(0)$ \\
\hline Periprocedural MI & $1(5)$ \\
\hline Distal embolization & $1(5)$ \\
\hline \multicolumn{2}{|l|}{ Follow-up: } \\
\hline Follow-up period & $3.2(1.2-5.3)$ \\
\hline ACS & $0(0)$ \\
\hline Stent thrombosis & $0(0)$ \\
\hline Repeat revascularization & $0(0)$ \\
\hline Stroke & $0(0)$ \\
\hline $\begin{array}{l}\text { Renal replacement therapy } \\
\text { (non-dialysis patients) }\end{array}$ & $0(0)$ \\
\hline Death & $1(5)$ \\
\hline \multicolumn{2}{|l|}{ Medications: } \\
\hline Aspirin & $20(100)$ \\
\hline Thienopyridine & $20(100)$ \\
\hline Anticoagulant & $9(45)$ \\
\hline$\beta$-Blocker & $18(90)$ \\
\hline$\alpha$-Blocker & $11(55)$ \\
\hline ACEI/ARB & $9(45)$ \\
\hline Calcium channel blocker & $11(55)$ \\
\hline Nitrate & $3(15)$ \\
\hline Statin & $14(70)$ \\
\hline Diuretic & $16(80)$ \\
\hline Oral hypoglycemic agent & $5(25)$ \\
\hline Insulin & $5(25)$ \\
\hline
\end{tabular}

Values are $n(\%)$, mean $\pm S D$ or median (Q1-Q3). $P C l$ - percutaneous coronary intervention, eGFR - estimated glomerular filtration rate, AKI - acute kidney injury, MI - myocardial infarction, ACS - acute coronary syndrome, ACEI/ARB angiotensin-converting enzyme inhibitor/angiotensin receptor blocker. 


\section{Discussion}

Zero-contrast $\mathrm{PCl}$ is a new promising approach in the prevention of AKI in patients with severe CKD. However, current evidence for its feasibility and safety is based on case reports and one retrospective study involving 31 patients [5-14]. Therefore, any new clinical data verifying this method provides valuable information on its effectiveness, which is paramount for its wider dissemination among the interventional community. Until now, zero-contrast techniques were employed only in stable patients, and subjects with acute coronary syndromes were not considered for such interventions [6]. This retrospective analysis summarizes the mid-term outcomes of zero-PCl carried out in a heterogeneous group of patients involving both stable subjects and unstable ones with acute coronary syndromes. The rationale for such an approach was to reduce renal function deterioration in a wide range of high-risk patients, e.g. in individuals with severe CKD who recently underwent standard primary $\mathrm{PCl}$ or experienced contrast-induced AKI. The estimated median probability of $\mathrm{AKI}$ in non-dialysis patients was $26 \%$ according to the Mehran risk score, but this risk was calculated with the assumption that contrast dye was not used - if these patients were treated with standard $\mathrm{PCl}$ using 100-200 $\mathrm{ml}$ of contrast medium, the median risk would increase to $57 \%$. Thanks to the zero-contrast approach, the factual risk of AKI was reduced to $10 \%$ and no patient required renal replacement therapy. Of note, two patients who previously developed contrast-induced AKI after standard coronary angiography and $\mathrm{PCl}$ did not experience AKI again after zero- $\mathrm{PCI}$.

There was one periprocedural "laboratory" myocardial infarction, yet without any clinical sequelae [17]. At the end of the procedure, due to legal issues, each patient obtained a small contrast volume (usually $5 \mathrm{ml}$ ) to document the final PCI result. Such a small contrast amount should not pose any relevant problem in terms of renal function, but it allowed us to recognize distal embolization in one patient with NSTEMI which was undetectable in IVUS, and consequently an adequate drug therapy was employed. During the median follow-up period of 3.2 (1.2-5.3) months, no patient experienced acute coronary event or required revascularization.

In the study group, there were 4 hemodialysis patients with preserved residual renal function. Three of these patients maintained their renal function, but in one patient the diuresis significantly dropped within some weeks after hospitalization. The latter patient had just started elective hemodialysis during the index hospitalization and other causes beside the coronary intervention might play a role in this loss of function. Namely, an initiation of hemodialysis by itself may suppress diuresis in some patients, which is associated with a number of factors, e.g. type of dialysis, hydration, congestive heart failure, diabetes and medications [18-20]. Therefore, it is unlikely that zero-PCI was the cause of the loss of renal function in this subject. In another hemodialysis patient, who underwent a 3-vessel zero- $\mathrm{PCl}$, the left ventricular function improved along with residual renal function and after some weeks the rate of dialysis was reduced. To the best of our knowledge, there is no report on zero- $\mathrm{PCl}$ in patients on chronic hemodialysis, but such an approach may help in preservation of their remaining renal function. Importantly, it has been demonstrated that the residual renal function is a prognostic and independent factor of quality of life, morbidity and survival in dialysis patients, and therefore every protective measure to preserve this function is worth considering [21]. Nevertheless, suitable prospective studies should address this strategy before it is recommended in clinical practice.

\section{Study limitations}

Several limitations of this study need to be acknowledged. This is a small and non-randomized study performed in a single center, which involved a heterogeneous group of patients, i.e. both stable and unstable ones, who faced a very high risk of AKI. Some patients were initially treated with standard procedures in the acute phase but zero-PCl was employed during further steps of revascularization as a staged intervention to protect their renal function. Although this makes the data non-homogeneous, it shows that the employment of zero- $\mathrm{PCl}$ at any stage of revascularization may bring benefit to patients with severe renal dysfunction. Each zero-PCl was done without contrast usage; nevertheless, at the end of the procedure usually one confirmatory injection of median contrast volume of 5 (3.5-9) $\mathrm{ml}$ was performed due to legal issues. Such a small amount of dye should not induce AKI, but this needs to be tested in further experiments. Finely, for more objective assessment of the real clinical benefit of the zero-contrast approach, randomized trials on large groups of patients are necessary. Despite all these limitations, the results of this study show that zero- $\mathrm{PCl}$ is feasible and is not associated with any specific complications.

\section{Conclusions}

This retrospective analysis shows that zero-contrast $\mathrm{PCl}$ is a safe and effective method to preserve renal function in patients with severe renal impairment and hemodialysis subjects. Moreover, it yields good clinical outcomes in mid-term observation. Since the procedure was based on IVUS imaging, it allowed us to optimize stent implantation with good procedural results, and consequently no stent thrombosis occurred during the follow-up. Thanks to the training program, the learning curve for the experienced operators was steep and there was no conversion to standard $\mathrm{PCl}$ in the study group. Therefore, if the patient's profile or clinical situation indicates that the risk of $\mathrm{AKI}$ is high, zero- $\mathrm{PCl}$ should be 
considered as a preventative solution - this also refers to dialysis patients with residual renal function.

\section{Conflict of interest}

The authors declare no conflict of interest.

\section{References}

1. Azzalini L, Spagnoli V, Ly HQ. Contrast-Induced nephropathy: from pathophysiology to preventive strategies. Can J Cardiol 2015; 32: 247-55.

2. Tsai TT, Patel UD, Chang TI, et al. Contemporary incidence, predictors, and outcomes of acute kidney injury in patients undergoing percutaneous coronary interventions: insights from the NCDR Cath-PCI registry. JACC Cardiovasc Interv 2014; 7: 1-9.

3. Mehran R, Aymong ED, Nikolsky E, et al. A simple risk score for prediction of contrast-induced nephropathy after percutaneous coronary intervention: development and initial validation. J Am Coll Cardiol 2004; 44: 1393-9.

4. Ohno Y, Maekawa Y, Miyata $\mathrm{H}$, et al. Impact of periprocedural bleeding on incidence of contrast-induced acute kidney injury in patients treated with percutaneous coronary intervention. J Am Coll Cardiol 2013; 62: 1260-6.

5. Nayak KR, Mehta HS, Price MJ, et al. A novel technique for ultra-low contrast administration during angiography or intervention. Catheter Cardiovasc Interv 2010; 75: 1076-83.

6. Ali ZA, Karimi Galougahi K, Nazif T, et al. Imaging- and physiology-guided percutaneous coronary intervention without contrast administration in advanced renal failure: a feasibility, safety, and outcome study. Eur Heart J 2016; 37: 3090-5.

7. Karimi Galougahi K, Zalewski A, Leon MB, et al. Optical coherence tomography-guided percutaneous coronary intervention in pre-terminal chronic kidney disease with no radio-contrast administration. Eur Heart J 2016; 37: 1059.

8. Karimi Galougahi K, Mintz GS, Karmpaliotis D, Ali ZA. Zero-contrast percutaneous coronary intervention on calcified lesions facilitated by rotational atherectomy. Catheter Cardiovasc Interv 2017; 90: E85-9.

9. Sacha J, Feussette P. How should I treat unprotected LM stenosis in a patient with ACS who develops a severe contrast-induced nephropathy after coronary angiography? https://www. pcronline.com/Cases-resources-images/Cases/EIJ-HSIT/Treatment-strategy-for-left-main-stenosis-in-patient-with-CIN?auth=true\#comments_area (Published, 06 December 2017)

10. Azzalini L, Hachinohe D, Regazzoli D, Colombo A. Ultra-low contrast percutaneous coronary intervention in patients with severe chronic kidney disease. EuroIntervention 2018; 14: 896-97.

11. Parviz Y, Fall K, Stone GW, et al. Imaging and physiology to guide venous graft interventions without contrast administration in advanced renal failure. J Invasive Cardiol 2017; 29: E163-5.

12. Azzalini L, Mitomo S, Hachinohe D, et al. Zero-contrast percutaneous coronary intervention guided by dextran-based optical coherence tomography. Can J Cardiol 2018; 34: 342.e1-3.

13. Sacha J. Marking wire technique for zero-contrast percutaneous coronary interventions. Adv Interv Cardiol 2018; 14: 204-5.

14. Sacha J, Feussette P. Zero-contrast percutaneous coronary intervention of saphenous vein graft in a patient with chronic renal failure. Adv Interv Cardiol 2018; 14: 309-11.

15. Herget-Rosenthal S, von Ostrowski M, Kribben A. Definition and risk factors of rapidly declining residual renal function in peri- toneal dialysis: an observational study. Kidney Blood Press Res 2012; 35: 233-41.

16. Sacha J, Gierlotka M, Feusette P, et al. Ultra-low contrast coronary angiography and zero-contrast percutaneous coronary intervention for prevention of contrast-induced nephropathy: step-by-step approach and review. Adv Interv Cardiol 2019; 15: 127-36.

17. Thygesen K, Alpert JS, Jaffe AS, et al.; ESC Committee for Practice Guidelines (CPG). Third universal definition of myocardial infarction. Eur Heart J 2012; 33: 2551-67.

18. Moist LM, Port FK, Orzol SM, et al. Predictors of loss of residual renal function among new dialysis patients. J Am Soc Nephrol 2000; 11: 556-64.

19. Kjaergaard KD, Jensen JD, Peters CD, Jespersen B. Preserving residual renal function in dialysis patients: an update on evidence to assist clinical decision making. NDT Plus 2011; 4: 225-30.

20. Lu W, Ren C, Han X, et al. The protective effect of different dialysis types on residual renal function in patients with maintenance hemodialysis: a systematic review and meta-analysis. Medicine (Baltimore) 2018; 97: e12325.

21. van der Wal WM, Noordzij M, Dekker FW, et al. Full loss of residual renal function causes higher mortality in dialysis patients: findings from a marginal structural model. Nephrol Dial Transplant 2011; 26: 2978-83. 